\title{
Infantile nystagmus syndrome: Broadening the high-foveation-quality field with contact lenses
}

\author{
Giovanni Taibbi ${ }^{1,4,5}$ \\ Zhong I Wang',3 \\ Louis F Dell'Ossol-3 \\ 'The Daroff-Dell'Osso Ocular Motility \\ Laboratory, Louis Stokes Cleveland \\ Department of Veterans Affairs \\ Medical Center and CASE Medical \\ School; ${ }^{2}$ Department of Neurology; \\ ${ }^{3}$ Department of Biomedical \\ Engineering, Case Western Reserve \\ University and University Hospitals \\ Case Medical Center, Cleveland, OH, \\ USA; ${ }^{4}$ Pediatric Ophthalmology and \\ Strabismus Unit, San Paolo Hospital, \\ Milan, Italy; ${ }^{5}$ University of Milan, Milan, \\ Italy
}

\begin{abstract}
We investigated the effects of contact lenses in broadening and improving the high-foveation-quality field in a subject with infantile nystagmus syndrome (INS). A high-speed, digitized video system was used for the eye-movement recording. The subject was asked to fixate a far target at different horizontal gaze angles with contact lenses inserted. Data from the subject while fixating at far without refractive correction and at near (at a convergence angle of 60 PD), were used for comparison. The eXpanded Nystagmus Acuity Function (NAFX) was used to evaluate the foveation quality at each gaze angle. Contact lenses broadened the highfoveation-quality range of gaze angles in this subject. The broadening was comparable to that achieved during $60 \mathrm{PD}$ of convergence although the NAFX values were lower. Contact lenses allowed the subject to see "more" (he had a wider range of high-foveation-quality gaze angles) and "better" (he had improved foveation at each gaze angle). Instead of being contraindicated by INS, contact lenses emerge as a potentially important therapeutic option. Contact lenses employ afferent feedback via the ophthalmic division of the $\mathrm{V}$ cranial nerve to damp INS slow phases over a broadened range of gaze angles. This supports the proprioceptive hypothesis of INS improvement.
\end{abstract}

Keywords: contact lenses, infantile nystagmus, visual function

\section{Introduction}

Infantile nystagmus syndrome (INS) is an ocular motor disorder characterized by involuntary oscillations that are typically bilateral, conjugate, uniplanar, horizontaltorsional, pendular or jerk with increasing velocity slow phases (CEMAS 2001). Current INS treatment relies on optical (eg, spectacles, contact lenses), medical (eg, baclofen, memantine), and surgical procedures (eg, extraocular muscle tenotomy and reattachment at the original or a moved insertion) and is aimed at improving vision and visual function of individuals affected by INS. It has been demonstrated that the extraocular muscle tenotomy procedure, which is embedded in all extraocular muscle movement procedures, broadens and improves the range of high-foveation-quality gaze angles in INS (Dell'Osso et al 1999; Hertle et al 2003, 2004; Wang et al 2006). Convergence has also been shown to broaden and improve the range of high-acuity gaze angles in INS (Serra et al 2006). Both therapeutic methods allow INS patients to see "more" (a wider range of high-foveation-quality gaze angles) and to see "better" (improved foveation at each gaze angle).

Contact lenses have been shown to improve visual acuity and to damp the nystagmus intensity (Sédan 1966; Abadi 1979; Allen and Davies 1983; Dell'Osso et al 1988; Golubovic et al 1989); however, their effects on foveation characteristics have not been documented. In this study, we carefully examined a subject to determine whether contact lenses are able to broaden and improve the range of high-foveation-quality gaze angles. In order to gain more insight into the mechanisms of contact lenses' effects on INS, we also compared the effects to those of convergence in the same subject. 


\section{Methods}

\section{Recording}

We used a digital video system (EyeLink II, SR Research, Mississauga, ON, Canada) for the recording. The system had a linear range of $\pm 30^{\circ}$ horizontally and $\pm 20^{\circ}$ vertically. System sampling frequency was $500 \mathrm{~Hz}$, and gaze position accuracy error was $0.5^{\circ}-1^{\circ}$ on average. The data from this system were digitized at $500 \mathrm{~Hz}$ with 16-bit resolution. Immediately after the subject's prescribed contact lenses were inserted, the EyeLink signal from each eye was calibrated with the other eye behind cover to obtain accurate position information; the foveation periods were used for calibration.

\section{Subject and protocol}

This study was approved by the local IRB and written consent was obtained from the subject before the testing. The subject was a calm 66-year-old male with INS; his nystagmus waveforms were well-documented previously and their characteristics representative of others with INS. Of particular importance was the data collected on this patient over a time span of 45 years using several types of infrared reflection, several types of magnetic search coil, and several types of high-speed digital video recording systems. That unique volume of data confirmed the static nature of his INS characteristics under similar recording conditions and allowed comparisons to be made from data sets taken at different times for different studies. The subject had a convergence "null" and wore either 7 PD base-out prisms added to his refraction of OD: $+3.00 \mathrm{~S}-2.50 \mathrm{C}$ ax 150 and OS: $+3.50 \mathrm{~S}-2.75 \mathrm{C}$ ax 20 or contact lenses with no added prisms (the latter were typically only worn several times a year for sports like skiing). The best-corrected visual acuity was 20/25 with prisms and 20/40+ with contact lenses. The insertion of contact lenses has never produced any feelings of dizziness or instability in this subject. Our paradigms use either small LED's or reflected laser spots which are not acuity limiting and yield the same ocular motor data with or without refraction. The subject was seated in a chair with a headrest and a chin stabilizer, far enough ( $>5$ feet) from the stimulus screen to prevent convergence effects. At this distance the target subtended less than $0.1^{\circ}$ of visual angle. The room light was turned off during the recording. The experiment consisted of five trials performed by the subject while wearing contact lenses; we allowed time between trials for the subject to rest. Specifically, we tested horizontal fixation targets from $30^{\circ}$ left gaze to $30^{\circ}$ right gaze, in $5^{\circ}$ steps. For comparison, we retrieved data from a previous study (Serra et al 2006) for the same subject, who performed the same trials without any refractive correction at far and at near with a convergence angle of $60 \mathrm{PD}$.

\section{Analysis}

All the analysis was performed in MATLAB environment (The MathWorks, Natick, MA) using OMLAB software (OMtools; available from http://www.omlab.org). Eye position was sampled directly; it was pre-filtered with a low-pass filter with the cutoff frequency of $20 \mathrm{~Hz}$ to reduce the noise while minimally affecting the foveation periods. Analysis was always done on the fixating eye. Segments with inattention or blinking were discarded.

The eXpanded Nystagmus Acuity Function (NAFX) was used as the outcome measure (Dell'Osso and Jacobs 2002). It is an extension of the previous Nystagmus Acuity Function (NAF) that consists of a mathematical function containing the following waveform parameters: foveation-period duration, standard deviations of main foveation periods and velocities, and number of cycles in a fixation interval. In the OMtools software, we use a graphical user interface for NAFX's data selection and calculation (details can be obtained from http://www.omlab.org/OMLAB_page/ Teaching/Using_NAFX.html). The NAFX provides an objective and repeatable measure of nystagmus foveation quality that accurately predicts the best-corrected visual acuity possible for subjects without afferent visual system defects, independently of the eye-movement recording system, the nystagmus type and waveforms (Dell'Osso and Jacobs 2002). In the absence of afferent deficits, the NAFX was shown to be highly correlated with measured acuity. We averaged the NAFX values if multiple ones were obtained at each gaze angle.

The longest foveation domain (LFD) (Serra et al 2006; Wang et al 2006) is defined as the range of the gaze angles in which the subject's NAFX stays above $90 \%$ of the NAFX gaze-angle curve peak value. LFD is a measure of the broadness of the NAFX gaze-angle curve, ie, the INS subject's high-foveation-quality field. The NAFX uses eye-movement data of foveation periods to both predict the best-corrected, potential visual acuity in the presence of INS and, coupled with a pre-operative acuity measurement, to estimate the post-operative measured visual acuity.

\section{Results}

The top panels (a, b, and c) of Figure 1 show the typical waveforms and their corresponding NAFX values at $-20^{\circ}$, $0^{\circ}$, and $25^{\circ}$. These panels were generated by the NAFX software program from fixation data at these gaze angles. 

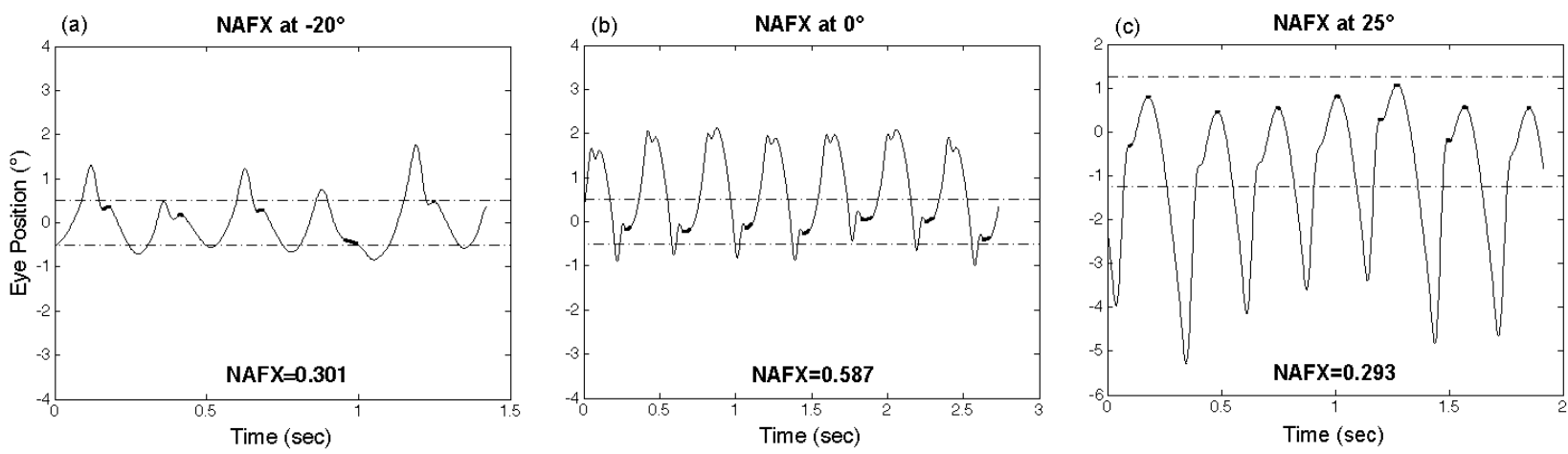

(d) NAFX, VERGENCE, CONTACT LENSES and GAZE ANGLE

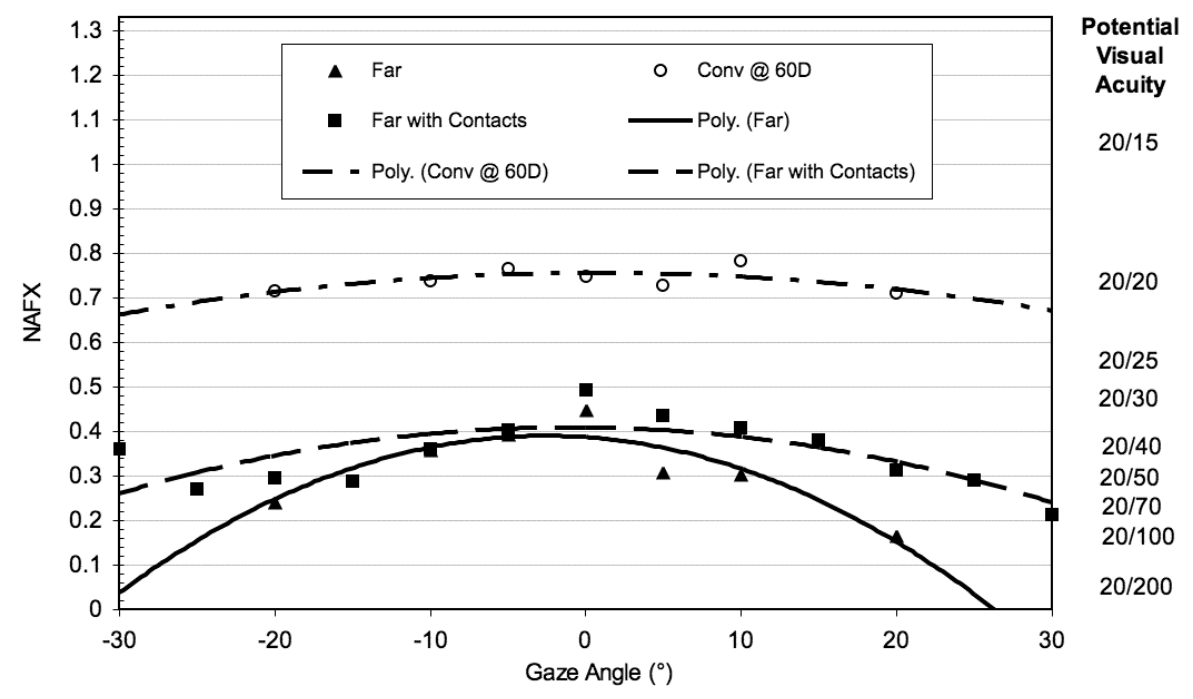

Figure I Fixation data (eye position vs. time) for the subject during far viewing with contact lenses at different gaze positions: $-20^{\circ}$ (a), $0^{\circ}$ (b) and $25^{\circ}$ (c). The corresponding NAFX values are also shown. Foveation periods were automatically thickened by the NAFX algorithm. The area between the dash-dotted lines represents the foveation position window used to calculate the NAFX. In (d), plots of NAFX vs. gaze angles for far viewing, far viewing with contact lenses, and while converged (60 PD). Fitted polynomial curves are shown. NAFX-correlated potential visual acuities are adjusted for the subject's age. Positive gaze angles indicate rightward gaze and "Conv" is convergence.

Upward eye movements in these panels denote a rightward eye movement. To assist comparison, the y-axis scales were set to be the same in all three panels. At the $-20^{\circ}$ gaze angle (a), the waveforms were of a pendular with foveating saccade type. The waveforms changed to pseudo pendular with foveating saccade when the eyes were straight ahead (b). At $25^{\circ}$ (c), the waveforms transitioned to jerk right. The nystagmus peak-to-peak amplitude at $25^{\circ}$ was the largest among all three gaze angles.

The NAFX value at $0^{\circ}$ was the highest; the foveation periods (thickened) were well lined up within a foveation position window of $\pm 0.5^{\circ}$ (dash-dotted lines). At $-20^{\circ}$, although the foveation periods were also in a foveation window of $\pm 0.5^{\circ}$, they had more position variation and higher slow-phase velocity. These factors contributed to a lower NAFX than that at $0^{\circ}$. At $25^{\circ}$, the position window was $\pm 1.25^{\circ}$, much larger than those at $-20^{\circ}$ and $0^{\circ}$. However, the NAFX value at $25^{\circ}$ was comparable with that of $-20^{\circ}$. The comparison of the NAFX values at the three gaze angles revealed that this subject had better foveation quality at primary position.

The bottom panel (d) of Figure 1 consists of three NAFX versus gaze-angle curves under different viewing conditions. The potential visual acuities corresponding to the NAFX values are listed on the right margin. The "Far with Contacts" curve is a second-order polynomial fit $\left(r^{2}=0.55\right)$ based on the NAFX values we obtained in this study. The "Far" $\left(r^{2}=0.86\right)$ and "Conv" $\left(r^{2}=0.44\right)$ curves were retrieved from a previous study. All three curves demonstrated peaks at $\sim 0^{\circ}$. When the subject was converging, the curve (dashdotted) was high and broad $\left(\mathrm{LFD}=50^{\circ}\right)$. When the subject was wearing contact lenses, the curve (dashed) was medium high and medium broad $\left(\operatorname{LFD}=35^{\circ}\right)$. These values are all greater than the $\pm 15^{\circ}$ range normally used before a head turn is employed. The solid curve (without contact lenses or converging) has the lowest peak and a narrow LFD of $15^{\circ}$. Comparing the dashed curve and the solid curve, the difference 
in NAFX at $+20^{\circ}$ and $-20^{\circ}$ is $\sim 0.15$ and 0.1 respectively, corresponding to a 1- and 2-line difference in Snellen acuities. Thus, although the two curves have similar peak values, the broadness is markedly better with the contact lenses. The two curves for "Far with Contacts" and "Conv" are virtually the same but at different NAFX/acuity levels. Convergence improves INS waveforms better than contact lenses, just as it does compared with the Kestenbaum procedure when both gaze-angle and convergence damping is present.

\section{Discussion}

One advantage of contact lenses over eyeglasses for INS patients (especially those with eccentric nulls) is that they allow fixation through the optical sweet spot at all times, thereby preventing distortion. Inspection of Figure 3 in the 1988 paper demonstrating that contact lenses damped INS over a range of gaze angles suggests that the curves were also broadened (Dell'Osso et al 1988). Our findings confirmed that suggestion by documenting a $133 \%$ (from LFD $=15^{\circ}$ to $\mathrm{LFD}=35^{\circ}$ ) broadening of the NAFX curve, which is directly correlated to improvements in visual acuity. Thus, contact lenses broaden the range of high-foveation-quality gaze angles in addition to any improvement in primary-position visual acuity they may provide. Figure 1 demonstrates that contact-lens broadening was equivalent to that of convergence but that the latter improved the NAFX to a greater extent. This subject did see "more" (a wider range of highfoveation-quality field) and "better" (improved foveation at each gaze angle) with contact lenses. This suggests that contact lenses do far more to improve visual function than has been previously appreciated and is a potentially important therapeutic option for INS patients.

The NAFX values shown in Figure 1 for $-20^{\circ}$ and $25^{\circ}$ are essentially equivalent, indicating that the corresponding potential visual acuities are also. However, the amplitude of the INS at $25^{\circ}$ is approximately four times greater. This reaffirms the poor correlation between nystagmus amplitude and potential visual acuity (Dell'Osso and Jacobs 2002) and illustrates why amplitude is only a reliable measure of cosmetic improvement, not visual function.

INS can be highly dependent on emotion and attention. However, using the same low-stress, non-acuity dependent recording conditions and protocols as we did in this study and all previous studies, if there is no additional sensory deficit acquired later in life, the baseline of INS does not change in the patient and the measurements of waveforms do not change either. We have over 40 years of eye-movement data from this subject, as well as multiple studies of other patients over years to decades, which demonstrate the stability of baseline INS over time. It was, therefore, justifiable to utilize historical data as the non-contact-lens control in this study. This study documented how contact lenses changed the subject's waveforms, especially compared to convergenceinduced changes; studying the broadening effects of contact lenses on more INS patients will determine the range of idiosyncratic foveation changes.

Contact lenses affect eye movements via afferent information carried on the ophthalmic division of the $\mathrm{V}$ (trigeminal) cranial nerve, not due to the mass of the lenses nor the refractive correction (Dell'Osso et al 1988). That was demonstrated by the use of topical anesthetics to limit the proprioceptive input from the contact lenses. Tenotomy has also been hypothesized to utilize proprioceptive information carried via the III, IV, and VI cranial nerves in addition to the $\mathrm{V}$ cranial nerve (Dell'Osso et al 1999; Hertle et al 2003, 2004). The absence of a proprioceptive effect on eye movements was the conclusion of an early study that only measured the effects on a specific class of ocular motor neurons (Keller and Robinson 1971). However, new studies have identified a second class, more amenable to taking part in a proprioceptive feedback loop (Büttner-Ennever et al 2001, 2002; Ugolini et al 2006). Specifically, a reduction of the steady-state muscle tension effectively reduces the muscle responsiveness to the small signals driving the slow phases (ie, small-signal gain reduction) (Wang et al 2006). The broadening effects we documented in this study provide evidence that the therapeutic effect of contact lenses on INS was not only due to their ability to correct refractive errors or to their optical properties (eg, lower spherical and chromatic aberrations than spectacles), but also due to their interference with the neurological mechanisms affecting the resulting nystagmus characteristics.

The broadening effect of contact lenses took place within the 30-minute time interval between insertion of the lenses and recording of the data. Previous studies of the effects of afferent stimulation of the $\mathrm{V}$ cranial nerve (pressure, touch, electric, vibration, and even air flow over the forehead - personal observation) have documented that this type of INS damping was immediate (ie, within milliseconds) (Dell'Osso et al 1988, 1991; Sheth et al 1995). Because contact lenses stimulate the same pathway, we conclude that their salutary effects in this study, as in the 1988 study (Dell'Osso et al 1988), also occurred instantaneously. This suggests that afferent stimulation of proprioceptive pathways modulates slow eye movements (eg, INS slow phases) in an 
on-line manner, not unlike a proprioceptive stretch reflex. We therefore, expect to find that contact lenses broaden the high-NAFX range of gaze angles in others whose INS is damped by them.

\section{Acknowledgments}

This work was supported in part by the Office of Research and Development, Medical Research Service, Department of Veterans Affairs (lfd, ziw) and Salmoiraghi e Viganò (gt).

\section{Disclosure}

This work was conducted in the Daroff-Dell'Osso Ocular Motility Laboratory, Louis Stokes Cleveland Department of Veterans Affairs Medical Center and CASE Medical School. There is no financial conflict of interest.

\section{References}

Abadi RV. 1979. Visual performance with contact lenses and congenital idiopathic nystagmus. Br J Physiol Optics, 33:32-7.

Allen ED, Davies PD. 1983. Role of contact lenses in the management of congenital nystagmus. Br J Ophthalmol, 67:834-6.

Büttner-Ennever JA, Horn AKE, Scherberger H, et al. 2001. Motoneurons of twitch and non-twitch extraocular fibres in the abducens, trochlear and oculomotor nuclei of monkeys. J Comp Neurol, 438:318-35.

Büttner-Ennever JA, Horn AKE, Graf W, et al. 2002. Modern concepts of brainstem anatomy. From extraocular motoneurons to proprioceptive pathways. In: Kaminski HJ, Leigh RJ (eds). Neurobiology of Eye Movements. From Molecules to Behavior - Ann NY Acad Sci 956. New York: NYAS; pp. 75-84.

CEMAS Working Group. 2001. A National Eye Institute-Sponsored Workshop and Publication on The Classification of Eye Movement Abnormalities and Strabismus (CEMAS). In: The National Eye Institute Publications (see www.nei.nih.gov). Bethesda, MD: National Institutes of Health, National Eye Institute.
Dell'Osso LF, Traccis S, Abel LA, et al. 1988. Contact lenses and congenital nystagmus. Clin Vision Sci, 3:229-32.

Dell'Osso LF, Leigh RJ, Daroff RB. 1991. Suppression of congenital nystagmus by cutaneous stimulation. Neuro Ophthalmol, 11:173-5.

Dell'Osso LF, Hertle RW, Williams RW, et al. 1999. A new surgery for congenital nystagmus: effects of tenotomy on an achiasmatic canine and the role of extraocular proprioception. J AAPOS, 3:166-82.

Dell'Osso LF, Jacobs JB. 2002. An expanded nystagmus acuity function: intra- and intersubject prediction of best-corrected visual acuity. Doc Ophthalmol, 104:249-76.

Golubovic S, Marjanovic S, Cvetkovic D, et al. 1989. The application of hard contact lenses in patrients with congenital nystagmus. Fortschr Ophthalmol, 86:535-9.

Hertle RW, Dell'Osso LF, FitzGibbon EJ, et al. 2003. Horizontal rectus tenotomy in patients with congenital nystagmus. Results in 10 adults. Ophthalmology, 110:2097-105.

Hertle RW, Dell'Osso LF, FitzGibbon EJ, et al. 2004. Horizontal rectus muscle tenotomy in patients with infantile nystagmus syndrome: a pilot study. J AAPOS, 8:539-48.

Keller EL, Robinson DA. 1971. Absence of a stretch reflex in extraocular muscles of the monkey. J Neurophysiol, 34:908-19.

Sédan J. 1966. Nystagmus et correction précornéenne de la myopie forte. Bull Soc Ophthalmol Fr, 66:1053-8.

Serra A, Dell'Osso LF, Jacobs JB, et al. 2006. Combined gaze-angle and vergence variation in infantile nystagmus: two therapies that improve the high-visual acuity field and methods to measure it. Invest Ophthalmol Vis Sci, 47:2451-60.

Sheth NV, Dell'Osso LF, Leigh RJ, et al. 1995. The effects of afferent stimulation on congenital nystagmus foveation periods. Vision Res, 35:2371-82.

Ugolini G, Klam F, Doldan Dans M, et al. 2006. Horizontal eye movement networks in primates as revealed by retrograde transneuronal transfer of rabies virus: differences in monosynaptic input to "slow" and "fast" abducens motoneurons. J Comp Neurol, 498:762-85.

Wang Z, Dell'Osso LF, Jacobs JB, et al. 2006. Effects of tenotomy on patients with infantile nystagmus syndrome: foveation improvement over a broadened visual field. JAAPOS, 10:552-60.

Wang Z, Dell'Osso LF, Zhang Z, et al. 2006. Tenotomy does not affect saccadic velocities: Support for the "small-signal" gain hypothesis. Vision Res, 46:2259-67. 
